\title{
Experimental Investigation on the Performance Evaluation of Solar Tracking Photovoltaic System
}

\author{
A. Kagilik*, S. Mousa, I.Enageem \\ Electrical and Electronic Department, Sabratha University, Sabratha, Libya \\ DOI: https://doi.org/10.21467/proceedings.2.21 \\ * Corresponding author email: as.kagilik@gmail.com
}

\begin{abstract}
The optimization of photovoltaic (PV) power generating system needs to be magnified by maximizing the solar radiation falling perpendicular on the PV module surface. The photovoltaic module can extract maximum power from solar irradiance by optimizing its orientation and its angle of inclination with the horizon. Therefore, it is recommended that for high efficiency and low loss of energy. PV module needs to be tilted at the correct angle. On the other hand, solar tracking system can be used to maximize the performance of the PV system, since solar tracking allows more energy to be produced because the solar array is able to remain aligned to the sun. The aim of this paper is to investigate experimentally the performance evaluation of solar tracking photovoltaic system. In this direction, an extensive experimental work at Faculty of Engineering, Sabratha-Libya to improve the output power of PV module by implementation of one axis tracking system. The project will include the design and construction of a microcontroller-based solar panel tracking system. The operation of the experimental model is based on DC motor intelligently controlled by a dedicated drive unit that moves PV panel according to the signals received from two simple but efficient light sensors. Practical comparison between fixed and tracking systems using identical PV modules is performed. It is found that the tracking system significantly improves the output power of PV module compared to the fixed PV panel under same climate conditions. Results indicated that the tracking system increases the output current by more than $60 \%$ compared with fixed PV panel, especially at early morning hours and before the sunset hours. This demonstrates, as expected, that the tracker photovoltaic system provides more efficient performance compared to the fixed photovoltaic system.
\end{abstract}

Keywords: solar radiation, Photovoltaic system, tracking system

\section{Introduction}

Recently, the demand of the renewable energy such as wind energy, solar energy, etc. has been increased dramatically around the world due to reduction in existing sources of fossil fuels and the growing concern regarding environment pollution. The conversion of solar light into electrical energy represents one of the most promising and challenging energetic technologies. Today, photovoltaics is considered as highly competitive technology and its world market is

(C) 2018 Copyright held by the author(s). Published by AIJR Publisher in Proceedings of First Conference for Engineering

Sciences and Technology (CEST-2018), September 25-27, 2018, vol. 1 .
This is an open access article under Creative Commons Attribution-NonCommercial 4.0 International (CC BY-NC 4.0) license, which permits any non-commercial use, distribution, adaptation, and reproduction in any medium, as long as the original work is properly cited. ISBN: $978-81-936820-5-0$ 
mainly fully developed including PV system components (mechanical/electrical). During the last decade, PV is applied at countless locations in many countries and has been implemented successfully with rapid falling installation costs and environments throughout the planet. The worldwide cumulative PV capacity reached about 303 GW by the end of 2016. During 2016, at least 75 GW PV capacity was added compared to 51 GW during 2015 with percentage increase up to $47 \%$. On the other hand, the global weighted average total installed cost of commissioned utility-scale (larger than 1 MW capacity) solar PV projects between 2010 and 2016 fell by $65 \%$, with the levelised cost of electricity (LCOE) falling by $67 \%$ over the period. In 2016, an average LCOE of around USD 0.12 per kWh, and a range of USD 0.05 per $\mathrm{kWh}$ to USD 0.35 per $\mathrm{kWh}$ was achieved according to region [1].

The maximum solar energy extracted from the solar phonons is affected by the amount of the collection of the sun radiation and the performance of PV power generating system is highly influenced by the incident solar radiation reaching the surface of the collector. Solar irradiation on PV modules varies with the modules position and the PV output power takes its maximum value when the solar radiation is perpendicular on the collector. Therefore, the optimization of PV module needs to be magnified by optimizing its orientation and its angle of inclination with the horizon. From our previous work, the strongly dependence of the maximum output power on the optimum inclination angle of the fixed PV solar module has been demonstrated and it has been found that the optimum tilt is different for each month of the year and the yearly average optimum tilt is approximately equal to the latitude of the site. Unfortunately, in spite of keeping the classical fixed PV panel in optimum position, the necessary optimum insulation for the maximum performance of PV module cannot be obtained during the whole year [2]. Thus an annually improving the output energy can be obtained by aligning the PV module with the sun such that the maximum radiation falls perpendicularly on the module surface, and the problem of loss can be avoided during the whole year.

Recent technology for optimization both the PV solar module orientation and angle of inclination and maximizing the PV performance is characterized by using the solar tracking system. Unlike the classical fixed PV panel, the function of the solar tracking device is following the sun trajectory such that the solar module is kept under optimum insolation for all positions of the sun. The sun tracking system approach must be equipped with essential features which are the azimuth tracking for adjusting the tilt angle of the surface of the PV array during changing seasons; and daily solar tracking for maximum solar radiation incidence to the PV array [3]. There are two main types of trackers, single axis and dual-axis, which usually operate using either a passive or active mechanism. It has been estimated that the tracking system increases the yield by $(30-60 \%)$ compared to stationary one. However, although the tracking systems strongly enhance the module efficiency, they increase the initial cost and complexity of the system and therefore they are still not practical solution for a smallscale application [4].

Proceedings of First Conference for Engineering Sciences and Technology (CEST-2018), vol. 1 
Experimental Investigation on the Performance Evaluation of Solar Tracking Photovoltaic System

To date, the performance of photovoltaic (PV) systems with different available technologies of solar trackers have been designed and executed by many workers. In this direction, numerous methods and mathematical models have been considered to validate the design methodologies. Many measurements setup including microcontroller-based solar panel tracking system, stepper motors and drivers, light intensity sensors, voltage regulation, physical construction, and software/system operation explanation have been used to obtain the optimum PV system performance under different climate conditions. Their analyses show an increase of solar irradiation upon a tilted system, azimuth tracker system, and dual axis tracker system as compared to the horizontal system $[4,5]$.

The aim of this paper is to investigate experimentally the performance evaluation of a single axis solar tracking photovoltaic system under Libyan climate conditions. The measurements are performed during the whole year and the optimized tracking system for four seasons are documented. Different preliminary results are presented in a number of graphs and table for a better interpretation. The proposed tracking method in this paper has been compared with conventional fixed method and good agreement with other investigators has been demonstrated.

\section{Solar Photovoltaic Tracking System}

\subsection{System description}

The main solar tracking PV system under investigation in this work is presented. The singleaxis solar tracking system consists of a PV panel rotating around a tilted shaft, under the action of a DC motor controlled with respect to the real Sun position estimated by means of two light intensity sensors. Depending on the orientation type solar trackers have a movement part based on a sun trajectory that updates the PV panel position according to the instantaneous solar irradiation. Therefore, the proposed solar tracking system was designed and executed so that specific technical requirements such as: optimum performance, operation reliability, movement simplicity are satisfied. Figure 1 shows the PV solar tracking system which designed and built in this work, while figure 2 illustrates the basic control circuit for a unipolar stepper motor or the complete hardware schematic of the system. As shown in the figures above, the solar tracking PV panels consists of moving parts and control elements, such as: PV module, DC motor, Light sensors, Limit Switch, relay and the Microcontroller. In this section, the background information on the main subsystems of the project was presented. The main part of this system is the PV module under measurements which is considered as a energy source. The second part of the system is the stepper DC motor. This motor is commonly used for precision positioning control applications and it was chosen to position the tracking sensor. The motor is characterized by brushless device, open loop positioning capability, good holding torque, excellent response characteristics and less circuit complicity.

ISBN: 978-81-936820-5-0 


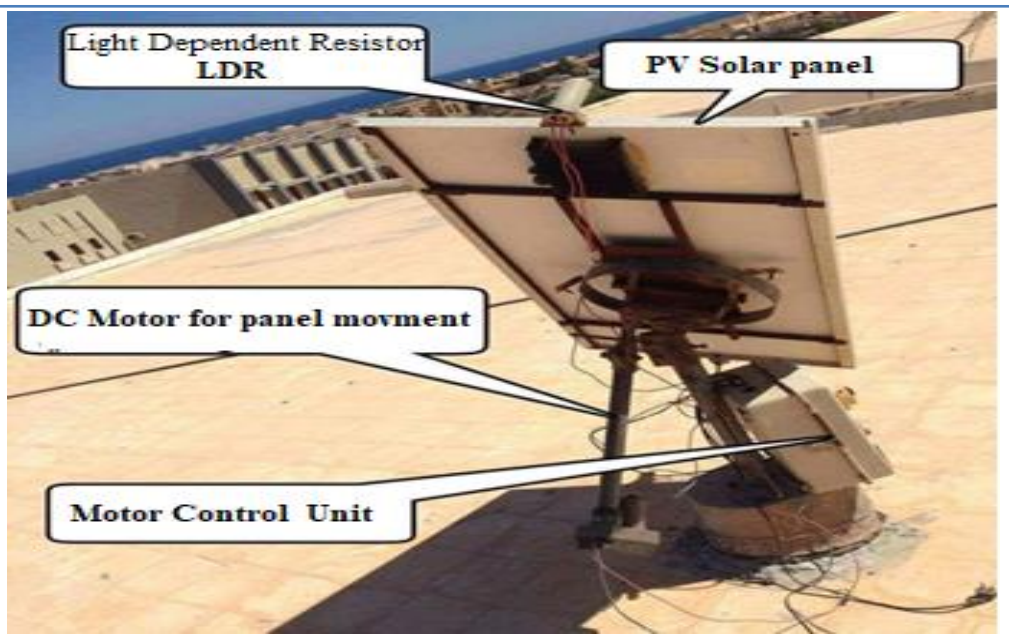

Figure 1: Solar tracking system

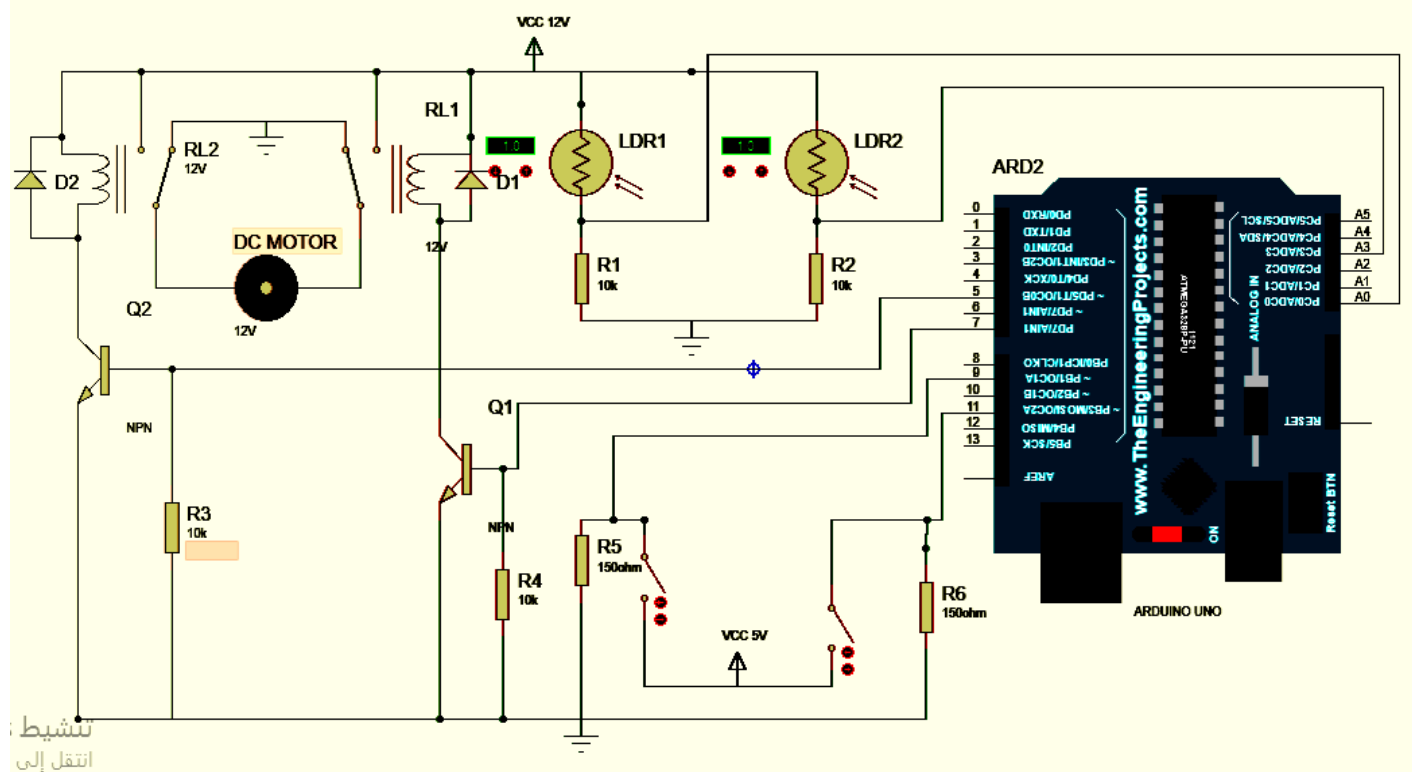

Figure 2: Hardware Schematic Diagram

The project consists of two reading of light sensor values which can control a motor to align with the greatest value which corresponds to the sun's position. Thus, these sensors allow an update of the PV panels position on a monthly or seasonal basis to obtain better PV performance and increase the global energy output of the system. Light sensors are among the most common sensor type. The simplest optical sensor is a photo resistor or Light Dependent Resistor (LDR) that the resistance of these sensors decreases with an increase in solar intensity. This is the least expensive and least complex type of light sensor.

Lastly, a microcontroller is provided to the control circuit. Since the project's focus is on embedded software control, the microcontroller is the heart of the system. The 
Experimental Investigation on the Performance Evaluation of Solar Tracking Photovoltaic System

microcontroller selected for this project is able to convert the analog photocell voltage into digital values and also provide output channels to control motor rotation. The ArduinoTM MEGA2560R3 was selected as it satisfies these requirements in addition to already being provided with the class lab kit. MEGA 2560 R3 is an Arduino compatible board with Microcontroller AT Mega 328, it's based on Arduino MEGA 2560 design. All Code, shield and IDE for Arduino MEGA 2560 R3 are also valid on BUONO MEGA 2560, some visible improvement on hardware make BUONO more flexible and easier use, and it's designed for projects that require more I/O lines, more sketch memory and more RAM with 54 digital I/O pins, 16 analog inputs. The Arduino Mega 2560R3 is programmed using the Arduino Software (IDE). The Arduino Programming language is easy to learn and understand since the Arduino IDE is linked with some software's such as: Max MSP processing and MATLAB.

\subsection{Algorithm for Control of Tracking System}

Figure 3 shows the flowchart of the tracking system control algorithm, while figure 6 illustrates the system protocol. As can be seen, the operation of PV tracking system is based on sensor method. This programming method, using Proteus software, is to track sun's trace by using photo sensors (LDR's) that is tracked through difference in amount of light. On the other hand, the photo resistor and stepper motor theory is discussed in order to provide a better understanding as to how they relate to the solar tracker.

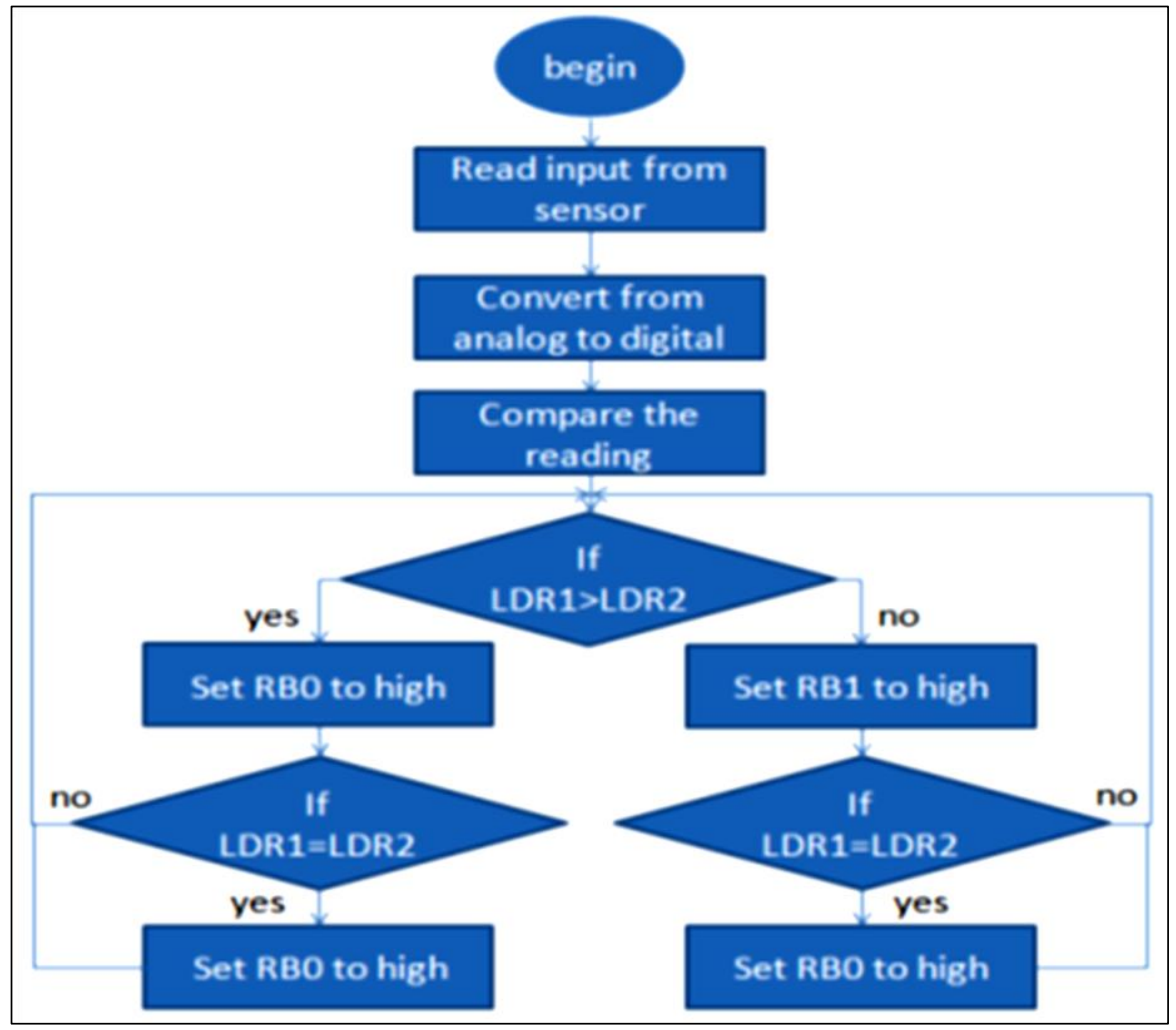

Figure 3: Flowchart of the tracking system control algorithm 


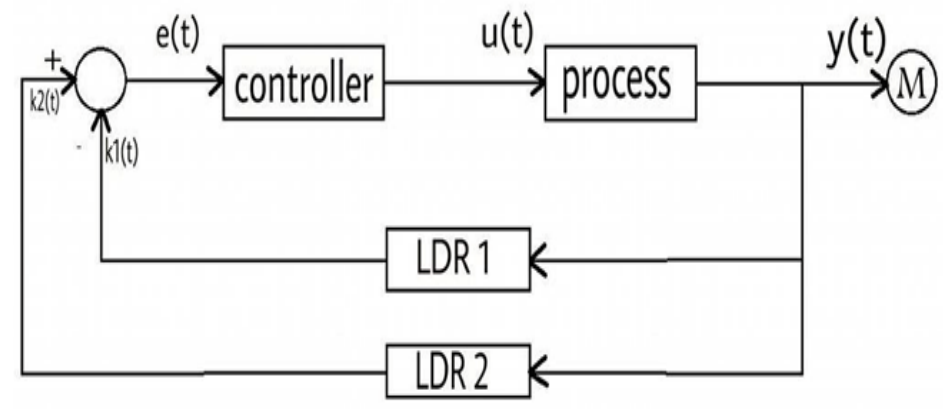

Figure 4: System control protocol

The error signal $e(t)$ is compared with the light intensity detected by the two LDRs as feedback signal. Therefore, the output error signal or the voltage difference will control to the movement direction of the PV panel, so as to bring it normally oriented to the incident sun light rays and thus to obtain a maximum conversion efficiency of light into electricity. The error signal is characterized by the difference between two sensors signals $\mathrm{K} 1(\mathrm{t})$ and $\mathrm{K} 2(\mathrm{t})$ through the relation given by:

$e(t)=k_{2}(t)-k_{1}(t)$

Where $k_{1}(t)$ and $k_{2}(t)$ are the first and second sensor signal, respectively. Depending on the light intensity imposed to the both sensors, there is a difference in the internal resistance value. That means when LDR1 receives much light that LDR2, then the internal resistance value of LDR1 is smaller than of LDR2 and hence this difference value will be received by the controller which works as a comparator that the output will send a signal to one of the transistors (processor) which works as On/Off switch, hence the transistor connect the current to the relay which control to the DC motor, as shown in figure 4. However, the DC motor that ensures the PV panel movement according to system protocol, as follows:

$e(t)=0$ no move

$e(t)>0$ right move

$e(t)<0$ left move

This process will be continued during the whole day until sun set. Therefore, the controller will receive a signal from the limit switch that the DC motor will move to its original position and stops its movement until the second day, and so on. This process is also happened in a very cloudy day. In this case, the motor control unit will readjust the solar panel position until the sun shines again to continue the desired protocol, but this phenomena is rarely happened in our climate here in Libya.

Proceedings of First Conference for Engineering Sciences and Technology (CEST-2018), vol. 1 
Experimental Investigation on the Performance Evaluation of Solar Tracking Photovoltaic System

\section{Experimental Investigation}

Experimental investigation was carried out in order to compare the performance of the solar tracking PV panel in comparison with a fixed PV panel of the same type. In this direction, an extensive experimental work at Faculty of Engineering, Sabratha-Libya was performed, to determine practically how solar PV tracking system can be optimized to extract the maximum power. Unfortunately, no long term ground global horizontal measurement stations were located near the site selected. However, it should be realized that all the data used in this work were based on the calculation of solar radiation in Sabratha city using MATLAB simulation software.

The photovoltaic performance of solar module was obtained by measuring the currentvoltage characteristics under natural climate conditions. The PV modules under investigation which were installed on the roof top of Electrical Engineering Department are TE750 TOTAL poly-crystalline commercially modules with capacity of $75 \mathrm{~W}$ and dimension of $31 \times 125 \mathrm{~cm}^{2}$ for each module. The typical specifications of the PV solar module at STC (AM1.5, 100W/ $\mathrm{m}^{2}$ at $25{ }^{\circ} \mathrm{C}$ ) is shown in Table I.

Table 1: Technical Data for Solar Module

\begin{tabular}{|l|l|}
\hline Electrical characteristics & Specifications \\
\hline Module type & TOTAL TE750 \\
\hline Number of Cells & 80 \\
\hline Typical Peak Power $\left(\mathrm{P}_{\max }\right)$ & $75 \mathrm{~W}$ \\
\hline Voltage at Peak Power $\left(\mathrm{V}_{\mathrm{mp}}\right)$ & $17 \mathrm{~V}$ \\
\hline Current at Peak Power $\left(\mathrm{I}_{\mathrm{mp}}\right)$ & $4.4 \mathrm{~A}$ \\
\hline Short Circuit Current $\left(\mathrm{I}_{\mathrm{sc}}\right)$ & $4.7 \mathrm{~A}$ \\
\hline Open Circuit Voltage $\left(\mathrm{V}_{\mathrm{oc}}\right)$ & $21.6 \mathrm{~V}$ \\
\hline Minimum Power $\left(\mathrm{P}_{\min }\right)$ & $70.1 \mathrm{~W}$ \\
\hline Minimum Pass Diode & $6 \mathrm{~A}$ \\
\hline Minimum Series Fuse Rate & $8 \mathrm{~A}$ \\
\hline
\end{tabular}

Unlike the classical fixed PV panel, the mobile one driven by solar tracker is kept under optimum insolation for all positions of the Sun, boosting thus the PV conversion efficiency of the system. The experimental procedure is characterized by measuring the output current, voltage and power produced from PV module that using single axis solar tracker system that automatically searches the optimum panel position with respect to the sun is compared with $30^{\circ}$ tilt angle fixed PV module to act as a comparison reference. It is documented from previous study that this tilt angle is found as the optimum angle for four seasons at this location [2]. However, to demonstrate that the tracking system was working in optimum conditions, a video camera has installed and observe the movement of the PV module if it can response to the sun position during the whole day. It was found that, fortunately, the system was working perfect and satisfactory results have been determined and documented. 


\section{$4 \quad$ Results and Discussion}

Solar irradiance is one of the most important parameter for evaluation of PV system performance. Based on site metrological data (latitude and longitude) of sabratah city, where the PV module characteristics measurements were taken, a simulation of solar radiation has be performed. The calculations have been done during the whole year. For comparison, two days in summer season where the sun shine duration is more than 7 hours (at about 1000 $\mathrm{W} / \mathrm{m}^{2}$ incident power) have been chosen. Figure 5 and figure 6 illustrate the average solar irradiance $\left(\mathrm{W} / \mathrm{m}^{2}\right)$ incident on $30^{\circ}$ tilted PV module. The calculations have been done for one day from the sun rise to sun set. As can be seen, it is found that the simulation results is in agreement with the data registered from metrological stations in Libya.

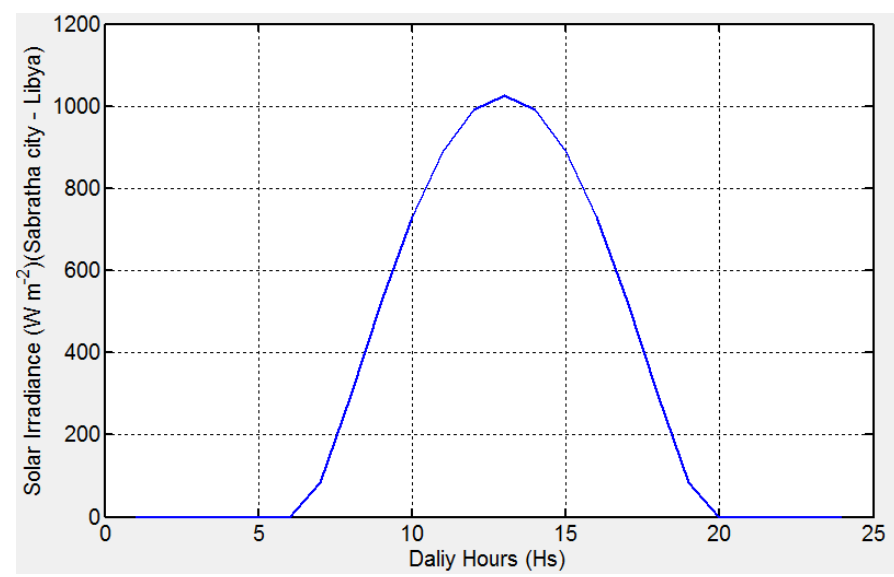

Figure 5: calculated solar radiation on tilted PV module using MATLAB simulation software (29.06.2017)

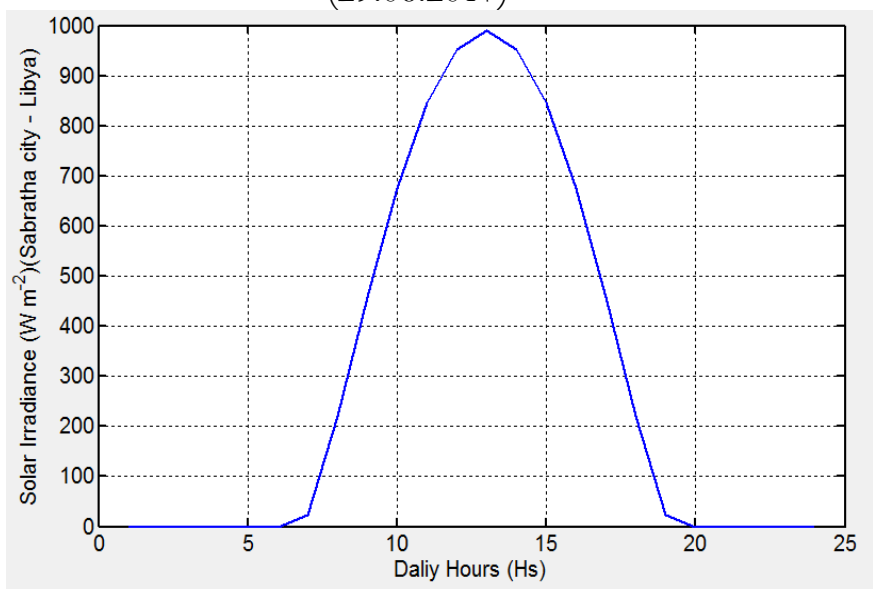

Figure 6: calculated solar radiation on tilted PV module (16.08.2017)

By measuring the current-voltage characteristics, the dependence of the photovoltaic performance on the solar irradiance has been investigated. The output power production of PV tracking module as compared to the fixed PV panel has been obtained, respectively. The

Proceedings of First Conference for Engineering Sciences and Technology (CEST-2018), vol. 1 
Experimental Investigation on the Performance Evaluation of Solar Tracking Photovoltaic System

measurements have been taken during four seasons and annual analysis has been documented. Since, the short circuit current $\left(\mathrm{I}_{\mathrm{sc}}\right)$ increases linearly with irradiance increasing, while the opencircuit voltage increases logarithmically with intensity, it is focused on the dependence of current on solar irradiance for both PV modules, due to the strongly dependence on radiation intensity [6]. The dependence of $\mathrm{I}_{\mathrm{sc}}$ on the solar radiation for fixed and tracking modules at selected two days are shown in figures 7 and 8 .

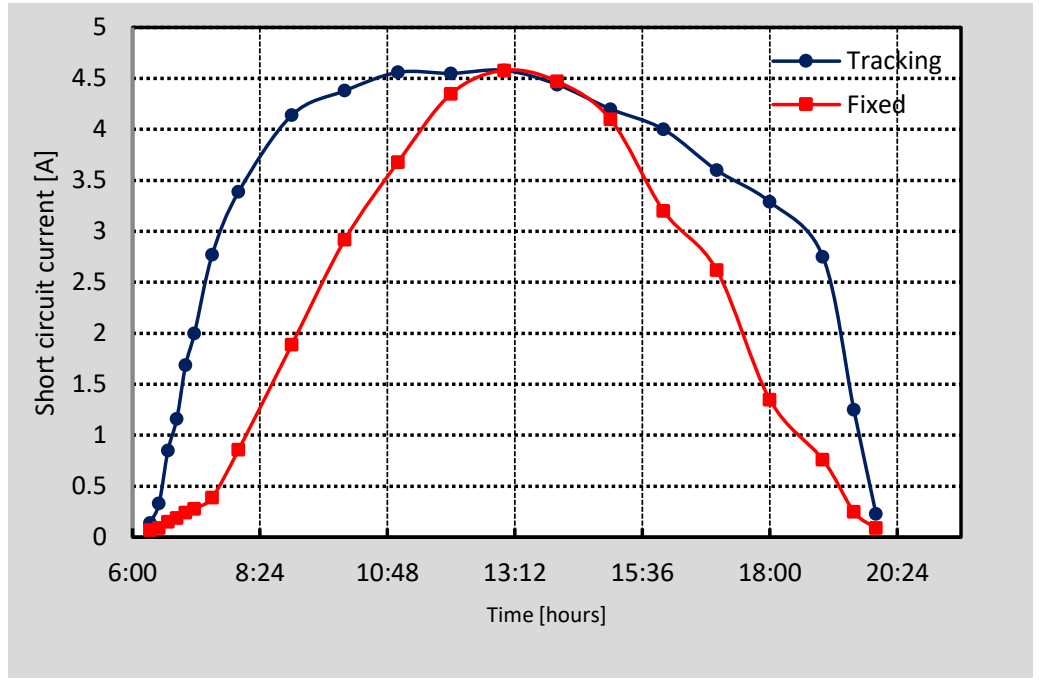

Figure 7: The dependence of the ISC on the solar irradiance (29.06.2017)

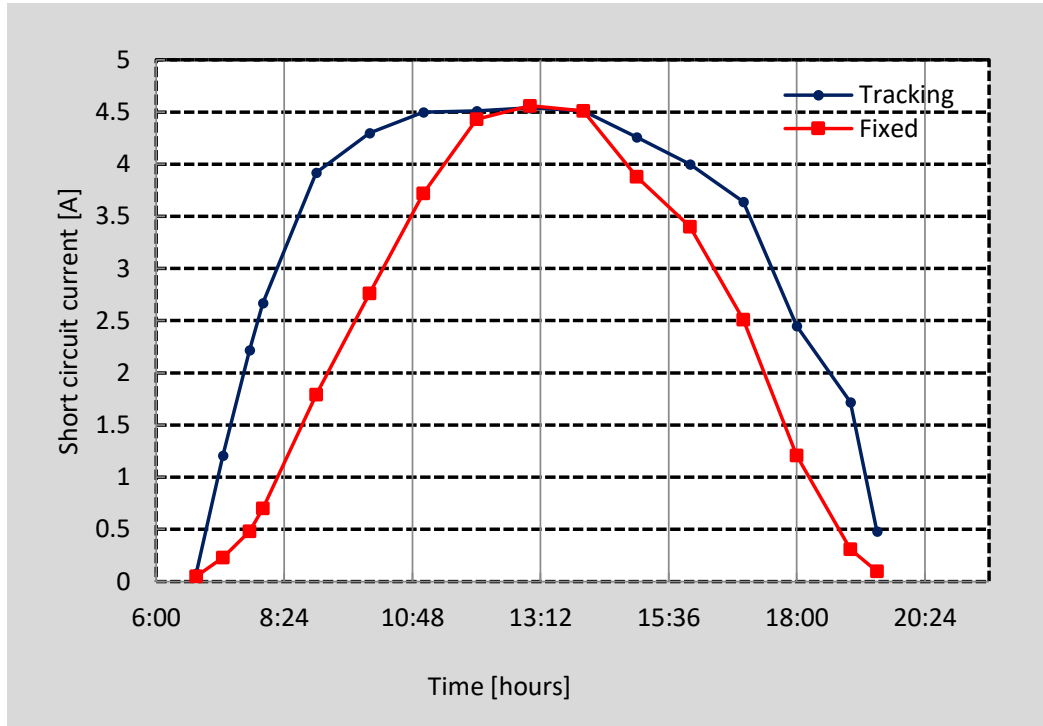

Figure 8: The dependence of ISC on the solar irradiance (16.08.2017)

As can be seen, the maximum current is obtained at the med day where the solar irradiance is maximum. On the other hand, the maximum performance obtained at optimum positions are very close to the module data specifications illustrated in table I. However, results show an increase in current of tracked panel as compared to the tilted fixed panel. This is because the maximum solar radiation is almost falling perpendicular on the tracked module surface during 
Kagilik et al., CEST-2018, AIJR Proceedings 2, pp.166-175, 2018

the whole day, which is not possible for the fixed PV panel [7]. The tracked module achieves higher performance at the morning and evening period than the fixed PV panel, reaching up to $60 \%$ improvement at this time period due to the more light intensity absorbance. It is observed that at midday almost both panels have same output and no significant difference in current because of the same tilt angles at this time and all the systems receive almost the same amount of irradiation approximately. Finally, according to our results, it is demonstrated that the single-axis tracking panel has the highest performance compared to the other fixed PV panel.

\section{Conclusions}

The sun tracking has an essential advantage that provides the maximum output energy with high accuracy against any variation of incident light from the sun or climatic conditions change compare to fixed PV module due to the surface of PV module is not perpendicular toward the sun at each time. In this work, an experimental study has been made to improve the PV system output performance through maintaining the module surface in optimum position for receiving maximum solar radiation. In this direction, single-axis solar PV tracking system has been designed and executed. To demonstrate of the feasibility and effectiveness of solar tracking system, an experimental investigation has been performed.

The current-voltage characteristics of the tracked PV module have been carried out and the dependence of the short circuit current on solar irradiance has been determined. From these measurements, it has been found that there is an significant improvement in the PV module performance and an enhancement in module current of more than $60 \%$ has been obtained compared to the tilted fix PV module, specially at the morning and evening time periods. This can be explained by that the tracked module surface almost remains under perpendicular irradiance receiving maximum radiation during the whole day.

\section{References}

[1] REN21, Renewables 2017, Global Status Report, PP. 63-71, Paris: REN21 Secretariat, ISBN 978-3-9818107-6-9.

[2] S. Mousa, I. Najem, O. Wehida, A. Khlifa, and A. Kagilik, "Optimization of Tilt Angle for Extracting Maximum Power from Photovoltaic Modules", Libyan International Conference on Electrical Engineering and Technologies (LICEET 2018), 4-6 March, 2018, Tripoli - Libya.

[3] Bill Lane, "Solar Tracker", EEC 517, Department of Electrical and Computer Engineering, Cleveland State University, April 30, 2008.

[4] Tiberiu Tudorache, Constantin Daniel Oancea, Liviu Kreindler, "Performance evaluation of a solar tracking PV panel", U.P.B. Sci. Bull., Series C, Vol. 74, Iss. 1, PP. 3-10, 2012, ISSN 1454-234x.

[5] Mostafa mehrtash, Guillermo quesada, Yvan dutil, Daniel rouse, "Performance Evaluation of Sun Tracking Photovoltaic Systems in Canada", 20th Annual International Conference on Mechanical Engineering-ISME2012, 16-18 May, 2012, School of Mechanical Eng., Shiraz University, Shiraz, Iran, ISME2012-2329.

[6] S. Buday, "Measuring irradiance, temperature and angle of incidence effects on photovoltaic modules in Auburn Hills, Michigan”, MSc Thesis, University of Michigan, August, 2011

[7] S. Lakeou, E. Ososanya, B.O. Latigo, W. Mahmoud, " Design of a Low-cost Solar Tracking Photo-Voltaic (PV) Module and Wind Turbine Combination System", University of the District of Columbia.

Proceedings of First Conference for Engineering Sciences and Technology (CEST-2018), vol. 1 